\title{
TROSIEME CONFERENCE REGIONALE ORGANISEE PAR KONRAD ADENAUER STIFTUNG A DAR ES SALAAM
}

\section{Troisième groupe de travail:}

\section{LES ASPECTS ENVIRONNEMENTAUX DU DEVELOPPEMENT ECONOMIQUE.}

Groupe n`3 : Par Professeur KALALA ILUNGA MATTHIESEN, Assistants MUMBA KAKUDJI MARTIAL, GUY-PROSPERE DJUMA, PATERNE MURHULA BATUMIKE, NESTOR NKURUNZIZA, LEONARD SEBUCENSHA ET ANTIDIUS KAITU

Le thème de notre groupe de réflexion, comme l'indique le titre de ce résumé, s'intitule : « Les aspects environnementaux du développement économique ».

Pour comprendre notre thème, nous avons procédé par la compréhension du vocable « développement durable ». D'après le rapport Brundtland de 1987 « Le développement est dit durable lorsqu'il est conçu de manière à assurer la pérennité du bénéfice pour les générations futures $» .^{1}$

Le développement doit être à la fois économiquement efficace, socialement équitable et écologiquement tolérable. Le social de la population doit être un objectif, l'économie un moyen et l'environnement une condition.

Par rapport à notre réflexion, la question majeure est de savoir si nos législations respectives contiennent des dispositions en faveur du développement durable.

La réponse à cette question si pertinente question est double :

- En premier lieu, il est apparu de l'analyse des législations concernées que celles-ci contiennent effectivement des dispositions protectrices de l'environnement. Il est possible de citer à titre illustratif l'article 49 de la constitution du Rwanda ainsi que la loi organique numéro 04/2005 du 08 avril 2005 portant modalités de protéger, sauvegarder et promouvoir l'environnement, c'est le cas également de l'article 35 de la constitution Burundaise, la loi sur l'environnement de 2004 en Tanzanie ainsi que l'article 53 de la constitution et la loi numéro 11/009 du 09 juillet 2011 portant principes fondamentaux relatifs à l'environnement pour ce qui est de la République Démocratique du Congo;

- En second lieu, il ressort toutefois un écart entre le prescrit de la loi et la pratique. Cet écart s'explique notamment par la mauvaise gouvernance, l'absence d'une administration environnementale efficiente et la corruption.

1 Lire Rapport Brundtland de 1987 pour les amples explications sur le vocable développement durable. 
Par ailleurs, nous saluons certaines pratiques courageuses dans la protection de l'environnement notamment :

- l'interdiction de l'usage ou la commercialisation des sacs en plastique au Rwanda,

- l'exigence d'une garantie financière qui couvrirait les dommages éventuels à l'environnement dans le cadre de l'exploitation minière en Tanzanie;

- l'obligation d'obtenir un permis avant d'abattre un arbre au Rwanda comme au Burundi;

- l'obligation de mener une étude d'impact environnemental préalablement à toute exploitation minière en RDC ainsi que l'interdiction d'octroyer le permis d'exploitation minière dans les zones protégées (parcs et terrains marécageux) et l'interdiction d'importer les véhicules fabriqués depuis un certain nombre d'années.

Eu égard à tout ce qui précède, nous préconisons ce qui suit :

- Lutter contre la corruption;

- promouvoir la bonne gouvernance dans le secteur environnemental;

- Insérer dans nos législations les dispositions relatives à la mise en œuvre de la responsabilité des entreprises dont les activités sont susceptibles de polluer l'environnement;

- Harmoniser les pratiques et les législations protectrices de l'environnement au niveau régional et sous-régional;

- Promouvoir l'implication des entités locales et la société civile dans la protection de l'environnement (voir par exemple l'édit provincial sur l'interdiction d'importation en sachet dans la province du Sud-Kivu (RDC);

- Promouvoir la sensibilisation et l'accès à l'information dans le processus de protection de l'environnement. 